\title{
Environmental Hardness of Pt-Ti-O gate Si-MISFET Hydrogen Gas Sensors from Siloxane, Humidity, and Radiation
}

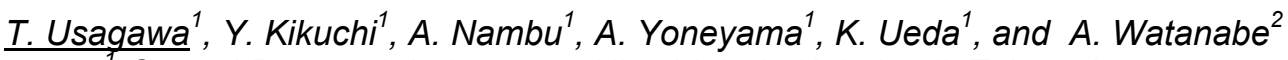 \\ ${ }^{1}$ Central Research Laboratory, Hitachi, Ltd., Kokubunji, Tokyo, Japan, \\ E-mail address : toshiyuki.usagawa.gv@hitachi.com \\ ${ }^{2}$ Hitachi Research Laboratory, Hitachi, Ltd., Hitachi, Ibaraki, Japan,
}

\begin{abstract}
Platinum-titanium-oxygen (Pt-Ti-O) gate Si-MISFET hydrogen gas sensors have been found to show large sensing amplitude $(\Delta \mathrm{Vg})$ of $624.4 \mathrm{mV}$ with $\sigma \Delta \mathrm{Vg}$ of $7.27 \mathrm{mV}$ for nine repeated measurements even under nitrogen-diluted $1.0 \%$ hydrogen gas, which are nearly the same values of $654.5 \mathrm{mV}$ with $\sigma \Delta \mathrm{Vg}$ of $3.77 \mathrm{mV}$ under air-diluted $1.0 \%$ hydrogen gas. The Pt-Ti-O sensor devices can work stably as hydrogen sensors up to about $300{ }^{\circ} \mathrm{C}$ and also show environmental hardness as follows: When nitrogen-diluted 10ppm HMDS (hexamethyldisiloxane) was exposed to the sensor FETs for 40 minutes at working temperature of $115{ }^{\circ} \mathrm{C}$, the sensing amplitude $(\Delta \mathrm{Vg})$ little changed within repeating errors before and after HMDS exposures. The variations of $\Delta \mathrm{Vg}$ between relative humidity of $20 \%$ and $80 \%$ are very small within $\pm 6 \%$ around $50 \%$ under the $40{ }^{\circ} \mathrm{C}$ atmosphere. The radiation hardness of developed hydrogen sensor chips from y ray $\left({ }^{60} \mathrm{Co}\right)$ and/or X-ray (Synchrotron Radiation) is sufficiently strong enough even at extremely high integral dose such as $1.8 \mathrm{MGy}$. It comes from the robustness of $\mathrm{PN}$-junction between drain and $\mathrm{p}$-well due to large device dimensions.
\end{abstract}

Key words: hydrogen gas sensors, Pt-Ti-O gates, Si-MOSFETs, radiation, humidity, siloxane

\section{Introduction}

Recently much attention has been paid to hydrogen gas sensors from various applications such as Fuel Cell Vehicles (FCV), atomic power plants, space transportation systems, resource exploration fields, and/or hydrogen energy systems, which require reliable hydrogensafety-monitoring sensor systems that can withstand so harsh environments.

Reliable catalytic gate structures are inevitably becoming necessary for possible candidates of Si-MOSFET hydrogen gas sensors. The Pt-Ti-O gate structures were invented to improve the reliability or to achieve stable sensing amplitude $\left(\Delta \mathrm{V}_{\mathrm{g}}\right)$ of $\mathrm{Pd}$ or Pt gate Si-MOSFET hydrogen gas sensors [1]. We have investigated device characteristics under severe harsh environments as common properties of Pt-Ti-O sensors in order to clarify the applicable limitations.

\section{Pt-Ti-O gate structures}

The Pt-Ti-O gate Si-MISFETs have unique gate structures composed of titanium and oxygen accumulated regions around platinum grains on top of a novel mixed layer of nanocrystalline TiOx and super heavily oxygendoped amorphous titanium formed on $\mathrm{SiO}_{2} / \mathrm{Si}$ substrates [1-4]. The novel structures of the sensors are typically realized by annealing $\mathrm{Pt}$ $(15 \mathrm{~nm}) / \mathrm{Ti}(5 \mathrm{~nm})$-gate Si-MOS structures in air at $400{ }^{\circ} \mathrm{C}$ for two hours. The oxidation process of the $\mathrm{Ti}$ layer is consistently explained by an oxidation model that the oxygen invasion into $\mathrm{Ti}$ layer comes from open air through $\mathrm{Pt}$ grain boundaries and at the same time $\mathrm{Ti}$ will evacuate into the Pt surface through Pt grain boundaries. During the course of this process, the invading oxygen will be balanced with the evacuating $\mathrm{Ti}$ so that the $\mathrm{Ti}$ layer keeps nearly the same thickness with the as grown states. Ti and oxygen remain around $\mathrm{Pt}$ grains named $\mathrm{Ti}$ and oxygen merged corridors [1-4].

The platinum grain boundaries act as the corridors for the invading oxygen from open air and for the evacuated titanium from the titanium layer toward the platinum surface. The formation of corridors is another origin of the large sensing amplitude due to broadening the pathway of hydrogen gas. 
One of the evidence for corridors of the Pt-Ti$\mathrm{O}$ gate MIS structures are shown by planar transmission electron microscope images (Fig. 1(b)), comparing with planar TEM image (Fig. 1(a)) of as grown $\mathrm{Pt} / \mathrm{Ti}$ gate structures.

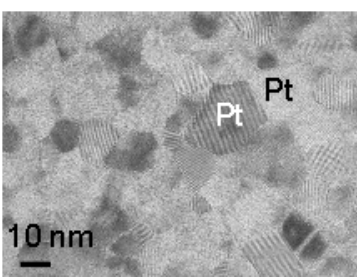

(a) Pt/Ti-gate

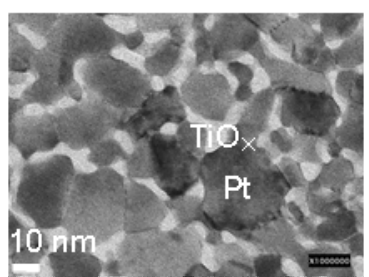

(b) Pt-Ti-O gate
Fig. 1. Planar TEM observation of as grown Pt/Tigate and Pt-Ti-O gate dummy structures.

A cross-sectional view of the Pt-Ti-O gate structures is schematically drawn in Fig.2, which has been confirmed by X-ray diffraction, Auger electron spectroscopy, and TEM energy dispersive X-ray spectroscopy [1-4]

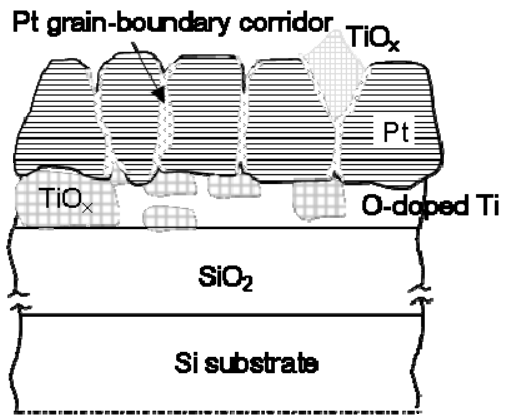

Fig. 2. Schematic cross-sectional view Pt-Ti-O gate structures.

\section{Basic Device Characteristics}

The Pt-Ti-O gate Si-MISFETs after 1000 ppm air-diluted hydrogen post-annealing at $115{ }^{\circ} \mathrm{C}$ for 10 minutes show reduced residual sensing amplitude $\Delta \mathrm{V}_{\text {gres. }}$. The sensing amplitude of $\Delta \mathrm{V}_{\mathrm{g}}$ is well described by a linear common logarithm between $100 \mathrm{ppm}$ and $1 \%$ of air-diluted hydrogen concentration $\mathrm{C}(\mathrm{ppm})$;

$$
\Delta \mathrm{V}_{\mathrm{g}}(\mathrm{V})=0.355 \mathrm{Log} \mathrm{C}(\mathrm{ppm})-0.610
$$

which is the best sensing range for most hydrogen-safety-monitoring sensor systems.

Si-MOSFET hydrogen gas sensors generally shows a Langmuir relationship between sensing amplitude $\Delta \mathrm{V}_{\mathrm{g}}$ and hydrogen concentration $\mathrm{C}$

$$
\Delta \mathrm{V}_{\mathrm{g}}=\Delta \mathrm{V}_{\mathrm{gmax}} \sqrt{\mathrm{C}} / \mathrm{C}_{0} /\left(1+\sqrt{\mathrm{C}} / \mathrm{C}_{0}\right)
$$

where $\Delta \mathrm{V}_{\text {gmax }}$ represents maximum sensing amplitude and central hydrogen concentration of $\mathrm{C}_{0}$ represents half occupied states of adsorption sites on the surface area of gate oxides [5]

The phenomenological logarithm fittings of experimental data such as eq. (1) are also prevailed. We have found that eq. (2) can be well described approximately by the following formula,

$$
\Delta \mathrm{V}_{\mathrm{g}}=\Delta \mathrm{V}_{\text {gmax }}\left[0.26 \log \left(\mathrm{C} / \mathrm{C}_{0}\right)+0.5\right]
$$

where both eqs. (2) and (3) are nearly overlapped for $0.1<\mathrm{C} / \mathrm{C}_{0}<10.0$ with two order of magnitudes. Equation (3) is similar to Padé approximation, but the coefficients of eq. (3) are determined to coincide with eq. (2) at $\mathrm{C} / \mathrm{C}_{0}=$ $0.1,1,10$. Two parameters of $\Delta \mathrm{V}_{\mathrm{gmax}}$ and $\mathrm{C}_{0}$ are $1365 \mathrm{mV}$ and $4338 \mathrm{ppm}$ derived by two eqs. of (1) and (3). The logarithm formula eq. (1) is well applicable for $440 \mathrm{ppm}<\mathrm{C}<4.4 \%$, which is a most suitable range for explosion prevention.

\section{Application to Harsh Environments}

\section{A. High Temperature Operation}

The operation temperature of the sensor devices has been confined to $115^{\circ} \mathrm{C}$. However, the upper limit of operation temperature as sensor devices will be crucial and should be clarified in case of so harsh environments. Figure 3 shows the temperature dependence of leak Ids currents $(\bullet) @ \mathrm{Vds}=1.5 \mathrm{~V}$ and Vgs= $-1.5 \mathrm{~V}$ for the fabricated FET with $\mathrm{Lg}=20 \mu \mathrm{m}$ and $\mathrm{Wg}=300 \mu \mathrm{m}$. Least square fitting formula is described in Fig. 3, where $\mathrm{x}$ and $\mathrm{y}$ represent $1000 / T$ and leak Ids. The typical sensing current levels are described by blue zone in Fig.3. The sensing Ids should be larger than the leak Ids current of the operation temperature. The activation energy of the exponential factor of the fitting formula is estimated to be 0.6558 $\mathrm{eV}$, which is nearly a half value of $\mathrm{Si}$ band gap.

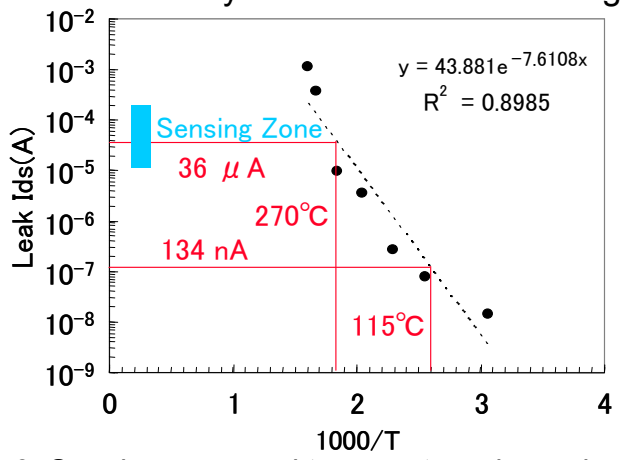

Fig. 3. Sensing zone and temperature dependence of leak lds current @ Vds=1.5V and Vgs=-1.5V.

The temperature dependence of sensing amplitude $\Delta \mathrm{V}_{\mathrm{g}}$ for air-diluted $0.1 \%$ hydrogen gas is shown in Fig. 4. The upper limit of hydrogen gas sensing is estimated to be about $300{ }^{\circ} \mathrm{C}$, in which leak Ids is around less than $100 \mu \mathrm{A}$, and is also justified from the allowable operation range of $\Delta V_{g}$ in Fig. 4 . 


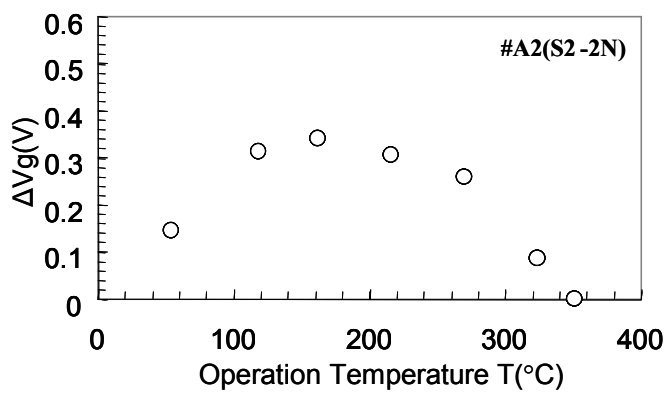

Fig. 4. Temperature dependence of sensing amplitude $\Delta V_{g}$ for air-diluted $0.1 \%$ hydrogen gas

The rapid decrease of $\Delta \mathrm{V}_{\mathrm{g}}$ above $300^{\circ} \mathrm{C}$ will be caused from rapid increase of leak lds or outgas contaminations in Fig. 4.

\section{B. Oxygen Free Environments}

One of the advantages for $\mathrm{Pt}-\mathrm{Ti}-\mathrm{O}$ gate $\mathrm{Si}$ MISFETs hydrogen gas sensors is capability of hydrogen gas detection in oxygen free environments as well as in air different from commercially available catalytic combustion or metal-oxide semiconductor type gas sensors.

We have shown an example of nine repeated response curves of our sensors at 7-minute intervals for two cases of nitrogen-diluted and air-diluted $1 \%$ hydrogen gas exposures. Both sensing amplitudes are nearly the same values; $624.4 \mathrm{mV}$ (in $\mathrm{N}_{2}$ ) and $654.5 \mathrm{mV}$ (in air) (Fig. 5). The standard deviations of $\Delta \mathrm{V}_{\mathrm{g}}$ for nine repeated measurements are very small values of $7.27 \mathrm{mv}, 3.77 \mathrm{mV}$, respectively.
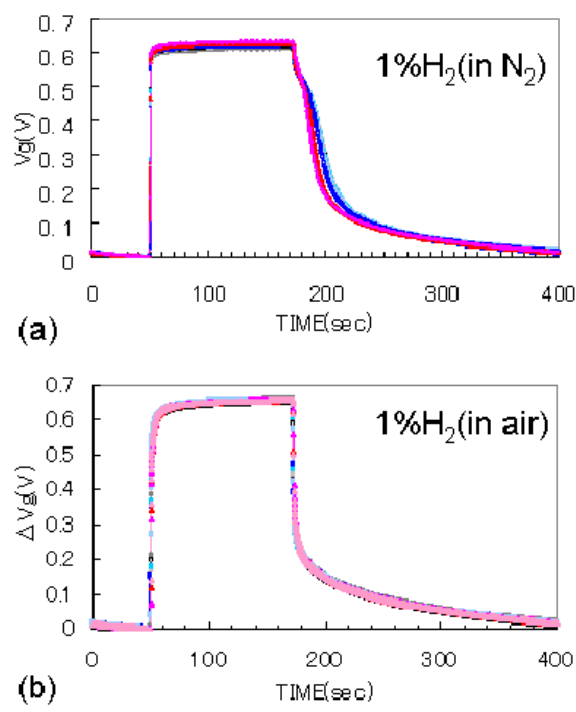

Fig. 5. Response curves of Pt-Ti-O sensors:

(a) nitrogen-, (b) air-diluted 1.0\% hydrogen gas

The small difference of $\Delta \mathrm{V}_{\mathrm{g}}$ between $624.4 \mathrm{mV}$ (in $\mathrm{N}_{2}$ ) $654.5 \mathrm{mV}$ (in air) is within variations of
$\Delta \mathrm{V}_{\mathrm{g}}$ over the wafers in stead of environmental gas difference.

\section{Siloxane \& Humidity}

The immunity from siloxane and humidity of hydrogen sensors is one of the key factors for short-term and long-term reliability. One of the data for immunity from HMDS is the response curves for nitrogen-diluted $0.1 \%$ hydrogen gas before and after nitrogen-diluted 10ppm HMDS exposures for $40 \mathrm{~min}$ (Fig.6). The residual sensing amplitudes observed after turning off hydrogen exposures are due to the non treatment of hydrogen post-annealing procedure:

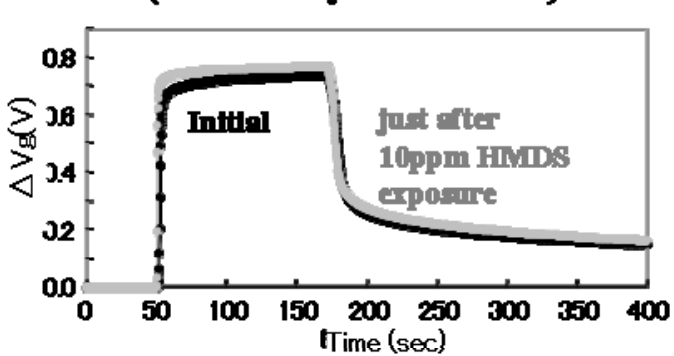

Fig. 6. HMDS test of $\triangle \mathrm{Vg}$ for nitrogen-diluted $0.1 \%$ $\mathrm{H}_{2}$ befoe and after HMDS exposure.

The aging tests of $V$ th from humidity and temperature are shown in Fig.7. The Vth keeps excellent stability for 55 days under high humidity conditions of $85^{\circ} \mathrm{C} 95 \%$.

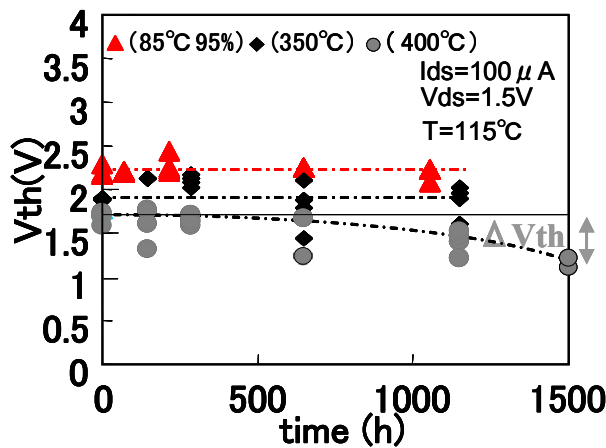

Fig. 7. Humidity and temperature aging tests for three conditions: $85{ }^{\circ} \mathrm{C} 80 \%$ humidity, $350^{\circ} \mathrm{C}$, $400^{\circ} \mathrm{C}$.

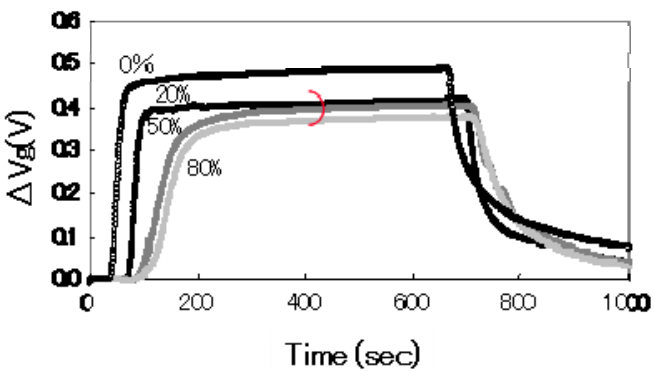

Fig. 8. Humidity test for four different relative humidity: 0\%,20\%,50\%, and 80\%@ 40 ${ }^{\circ} \mathrm{C}$. 
Figure 8 shows the response curves for $0.1 \%$ air-diluted hydrogen gas show very small variations, $\pm 6 \%$, of $\Delta \mathrm{V}_{\mathrm{g}}$ under four humidity conditions; dry, 20, 50, and $80 \%$ relative humidity @ $40^{\circ} \mathrm{C}$ by the corrections due to variations of residual sensing amplitude $\Delta \mathrm{V}_{\text {gres }}$.

\section{Radiation.}

Electric apparatus such as microprocessors or solid state image sensors are well recognized to have serious errors in their functions after rather small integrated radiation such as 100 10,000Gy. The radiation tolerance of electric apparatus should be kept under $2 \mathrm{M}$ Gy in severe accidents of atomic plants.

There are a lot of works about radiation effects on Si-MOSFETs for the last 50 years. However, device dimensions and/or the working conditions of hydrogen sensor devices are much different from hitherto-analyzed SiMOSFETs. We have analyzed the radiation effects on the various Pt-Ti-O gate Si-MISFETs by using y ray $\left({ }^{60} \mathrm{Co}\right)$ and/or X-ray (Synchrotron Radiation:Spring-8 \& Photon Factory, KEK)

One of the typical I-V characteristics of Pt-TiO gate Si-MISFETs is shown in Fig.9 before and after $\mathrm{Y}$-ray radiation with heavy integral dose of $1.785 \mathrm{~Gy}$ and dose rate of $5000 \mathrm{~Gy} / \mathrm{h}$. The leak Ids current @ Vgs=-1.5V and $\mathrm{Vds}=1.5 \mathrm{~V}$ is not suffered from such heavy irradiation. It means the robustness of $\mathrm{PN}$ junction between drain and $p$-well due to large device dimensions ; $\mathrm{Lg}=20 \mu \mathrm{m}$ and $\mathrm{Wg}=300 \mu \mathrm{m}$. The S-factor around sub-threshold regions is influenced by irradiation, however, it is not fatal to hydrogen gas sensing from practical point of view.

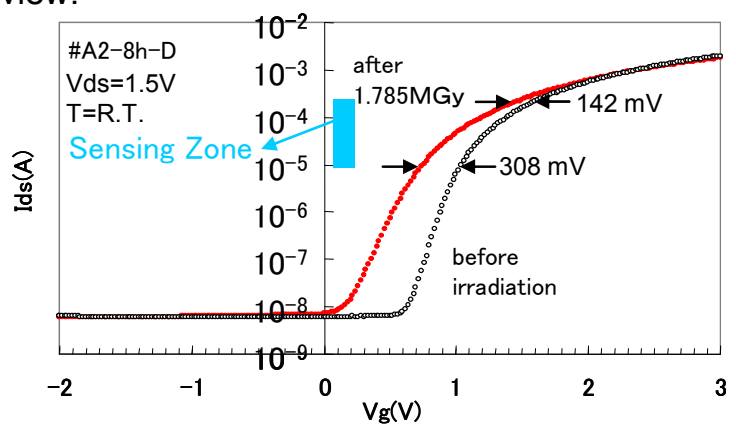

Fig. 9. I-V characteristics of Sensor FETs at room temperature before and after $y$-ray $\left({ }^{60} \mathrm{Co}\right)$ : $1.785 \mathrm{MGy}, 5000 \mathrm{~Gy} / \mathrm{h}$ for $357 \mathrm{~h}$. Blue region is a typical sensing zone.

Moreover, we have investigated the irradiation effects on the Pt-Ti-O gate SiMISFETs with wide range of integral dose, 100 Gy - 80M Gy, and also three energy levels of 1.33/1.17Mev, $115 \mathrm{kev}$, and $10 \mathrm{kev}$. Integral dose levels are normalized by a circle area with radius $\mathrm{r}=300 \mu \mathrm{m}$ for sensor FET area. The degradation of field effect mobility of inversion layers in Pt-Ti-O gate MISFETs is shown as the ratio of the mobility before and after irradiation at room temperature in Fig.10. In case of ultra high dose regions, the mobility degradation ratio is around 0.3 so that the Ids currents at socalled saturation region also reduce up to 0.3 . This large degradation of lds at saturated region surely causes fatal effects on their functions of microprocessors or image sensors.

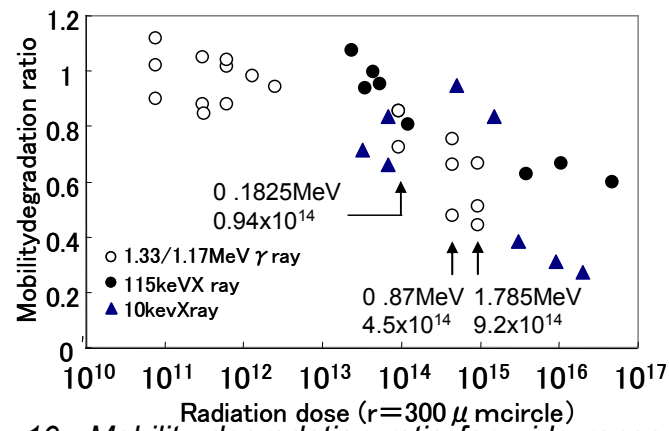

Fig. 10. Mobility degradation ratio for wide range of irradiation with three different photon energy.

\section{Conclusions}

Environmental hardness has been found unexpectedly as one of the advantages of Pt-Ti$O$ gate Si-MISFETs hydrogen gas sensors such as possible sensing to high temperature $300{ }^{\circ} \mathrm{C}$ or radiation hardness up to high integral dose of $2 \mathrm{M}$ Gy - 80M Gy. The sensor will be also expected to show strong robustness against severe poisoning or degradation from siloxane and humidity.

\section{Acknowledgements}

This work was supported in part by the New Energy and Industrial Technology Development Organization (NEDO), and also SPring-8 with the approval of the Japan Synchrotron Radiation Research Institute (JASRI). The sensor chips were fabricated by Takasaki Works, Renesas Electronics Corporation.

\section{References}

[1] T. Usagawa and Y. Kikuchi, J. Appl. Phys. 108, 074909, 2010.

[2] T. Usagawa and Y. Kikuchi, J. Appl. Phys. 110, 074515, 2011.

[3] T. Usagawa and Y. Kikuchi, Sens. Actuators B 160, pp.105-114, 2011.

[4] T. Usagawa and Y. Kikuchi, Jpn. J. Appl. Phys. 51, 024101, 2012.

[5] Y. Morita, K. Nakamura, and C.Kim, Sens. Actuators B 33, pp.96-99, 1996. 